\title{
Management of Ectopically Erupted Maxillary Canine by Segmented T-Loop and Orthodontic Mini-Screw: A Case Report
}

\author{
Ali Alhaizaey ${ }^{1, *}$ and Asma AlMousa ${ }^{2}$ \\ ${ }^{1}$ Orthodontic and dentofacial orthopedic consultant, King Faisal Medical City for Southern Region, Abha, Saudi Arabia \\ ${ }^{2}$ Orthodontic Resident, Department of Orthodontics, College of Dentistry, King Khalid University, Abha, Saudi Arabia
}

*Corresponding author: Ali Alhaizaey, Orthodontic and dentofacial orthopedic consultant, King Faisal Medical City for Southern Region, Abha, Saudi Arabia, E-mail: Ahalhayzaee@kfmcity.med.sa

Received: 21 Dec, 2020 | Accepted: 11 Jan, 2021 | Published: 15 Jan, 2021

Citation: Alhaizaey A, AlMousa A (2021) Management of Ectopically Erupted Maxillary Canine by Segmented T-Loop and Orthodontic Miniscrew: A Case Report. Int J Dent Oral Health 7(2): dx.doi.org/10.16966/2378-7090.350

Copyright: (C) 2021 Alhaizaey A, et al. This is an open-access article distributed under the terms of the Creative Commons Attribution License, which permits unrestricted use, distribution, and reproduction in any medium, provided the original author and source are credited.

Keywords: Mini-Screw; Segmented T-Loop; Ectopic canine

\section{Introduction}

The ectopic eruption is a deviation from the normal eruption pattern and may occur in permanent teeth for mean reasons, including trauma, infections, cysts, a supernumerary tooth, and crowded dentition [1]. The management of ectopically erupting teeth depends on the correction of established etiological factors, which may include the extraction of retained primary teeth or the removal of cysts or pathologies that are causing the deviated pattern, followed by orthodontic treatment with anchorage control [2]. Failure to treat ectopic eruption can result in a loss of arch length and malocclusion.

The success of orthodontic treatment generally relies on the anchorage protocol that is planned for a particular case. The use of extra-oral appliances like headgear is dependent on the patient's cooperation. Conventional intra-oral anchorage methods like the transpalatalarch and the Nance arch help in reinforcing the molars but they do not provide absolute anchorage $[3,4]$.

Dental implants, mini plates, and modified fixation screws provide bone anchorage in orthodontic treatment $[5,6]$. Recently, mini-screws have become popular because they are easier to insert and remove $[3,4]$.

The incorporation of mini-screws in the orthodontic field has allowed for more predictable anchorage control, increased the ability to correct severe skeletal and dental discrepancies, and prevented untoward tooth movement. The application of mini-screws in various clinical situations demanding movement of either a single tooth or a segment of teeth has been largely successful with ease and minimal invasiveness upon insertion and removal.

The mini-screws placed at the interdental alveolar bone can deliver forces directly to the tooth or arch-wire, eliminating the need for additional connectors. This versatility of mini-screws can be very helpful, especially for posterior segment control. This case report describes an unusual modality of using a mini-screw to achieve multiple orthodontic results in a compromised orthodontic case.

\section{Case Report}

A 17-year-old female patient presented to the orthodontic clinic with a chief complaint of severe crowding in the upper arch. She exhibited a convex profile, an obtuse nasolabial angle, an asymmetrical face due to mandibular deviation to the right, and a shifted upper midline to the left in relation to the facial midline (FML).

The upper arch was severely crowded with the left canine completely blocked out labially, and the lower arch showed moderate crowding.

The molars had a Class I relationship, the right canine had a Class I relationship, and the left canine was non-applicable. Vertically, she had reduced overbite (Figure 1). The clinical examination revealed a discrepancy between the centric relation (CR) and centric occlusion (CO) due to premature contact in the posterior teeth. In CR, when the mandible is guided to the most retruded unstrained position of the mandibular condyle within the temporomandibular joint (TMJ), the upper dental midline (UML) and lower dental midline (LML) coincided with each other, and at the same time, both of them deviated to the left side in relation to the FML. Also, the patient has a posterior crossbite and anterior open bite existed when the mandibular arch is guided to CR. However, in $\mathrm{CO}$, when the patient is asked to bite down into maximum intercuspation position, the mandibular arch deviates to the right side and the lower dental midline (LML) becomes centered with the patient's FML (Figure 2).

\section{Treatment goals}

The objectives of treatment were to achieve Class-I molar and canine relationships, achieve an ideal overjet and overbite, resolve to the crowd and posterior crossbite as well as resolving of jaw deviation and achieve fairly symmetrical facial proportions with upper and lower coincident dental midlines. 

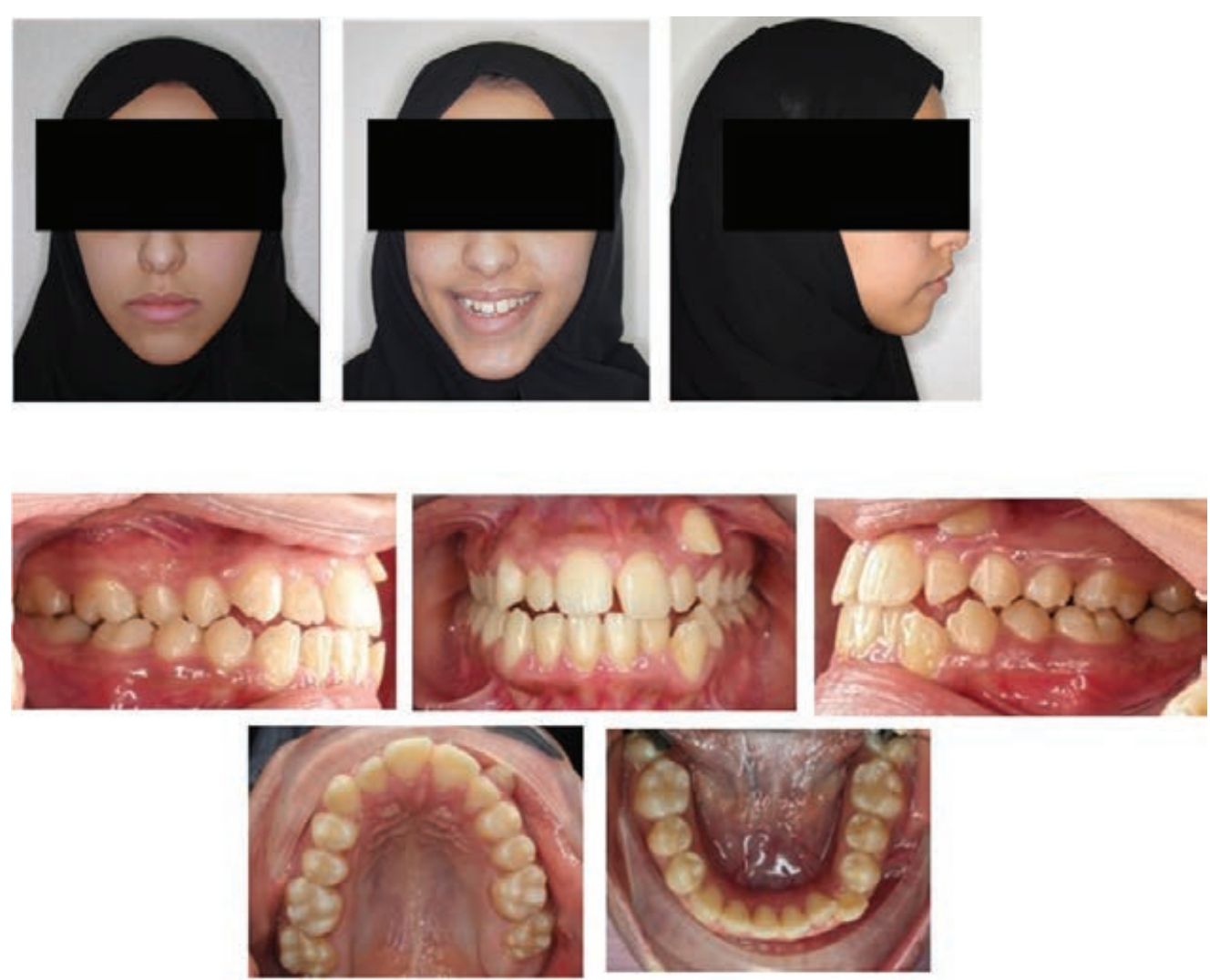

Figure 1: Pre-treatment intra-oral and extra-oral photographs.
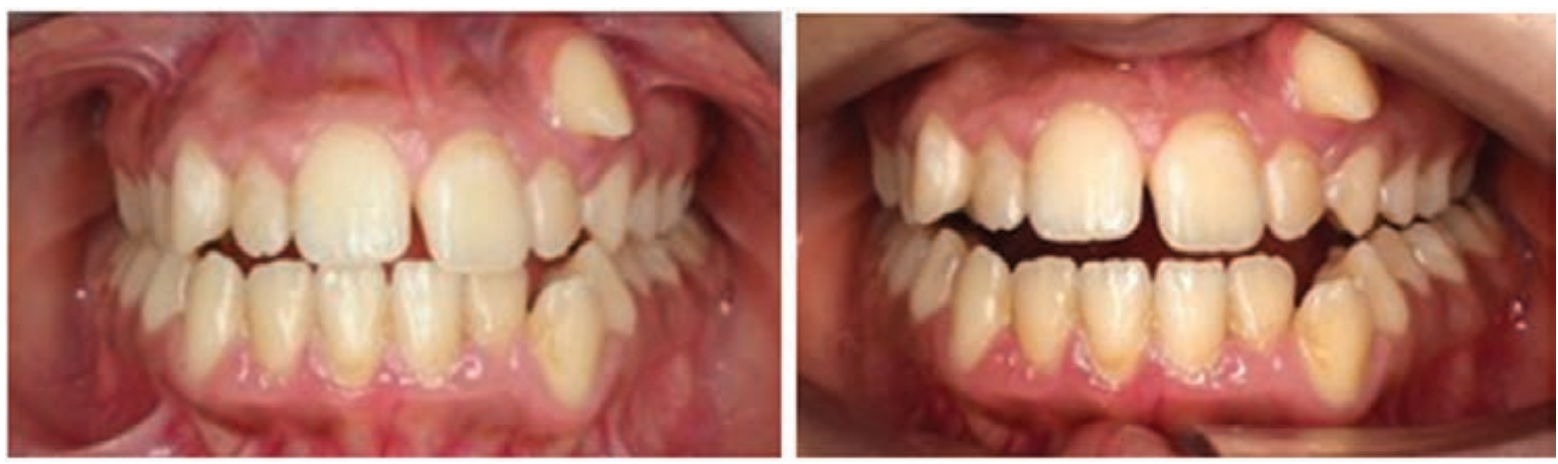

Figure 2: CR-CO discrepancy due to premature contact in posterior teeth. Here with patient in centric relation posterior cross bite and anterior open bite existed when mandibular arch is guided to (CR).

\section{Treatment plan}

The treatment plan was to extract upper and lower first premolars with mini-screw insertion in the upper left side to properly control the upper left canine movement. The upper and lower first premolars on the left side were extracted to relieve crowding whereas the upper and lower first premolars on the right side were extracted to fix upper and lower dental midlines. A quad helix appliance is also planned for maxillary arch expansion and resolving of posterior crossbite and mandibular deviation.

\section{Treatment progress}

After extraction of the upper and lower $1^{\text {st }}$ premolars, treatment was initiated by insertion of mini-screw (Ormco, $10 \mathrm{~mm} \times 2 \mathrm{~mm}$ ) between teeth \#25 and \#26 in the upper left area. A segmented T-loop (Readymade, $0.016 \times 0.022$ ) was used and extended from the mini-screws to the ectopically erupted upper left canine. The segmented T-loop was activated by pulling the wire from the mini-screws and then cinching it back to the mini-screw. A V-bend was added on the horizontal arm of the T-loop to counteract the moment from the force generated during activation of the T-loop and make the root upright to achieve bodily movement of the canine (Figure 3 ).

At the same time, the quad helix was activated and inserted to expand the maxillary arch (Figure 4). After adequate expansion was achieved, the quad helix alliance was maintained for additional 


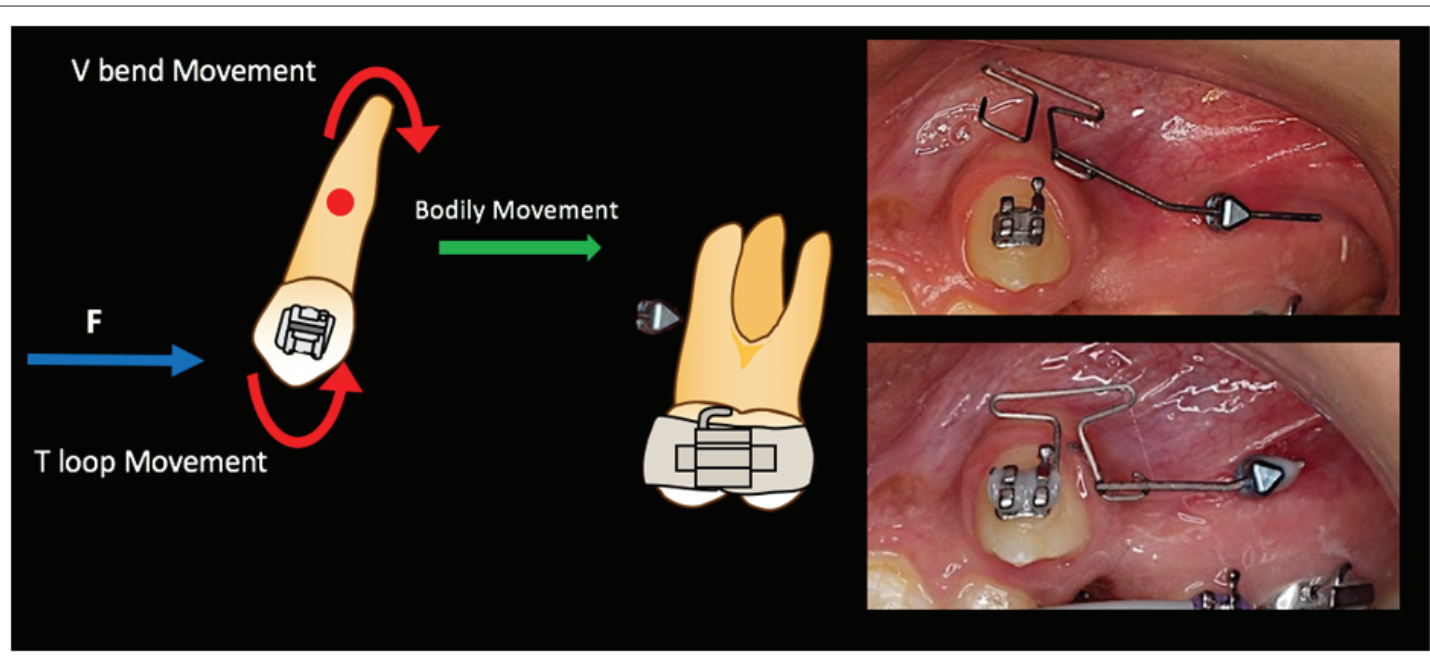

Figure 3: Mini-screw insertion between \#25 and \#26 and segmented T-loop activated and cinched back with V-bend in the arm to counteract the moment of canine retraction.

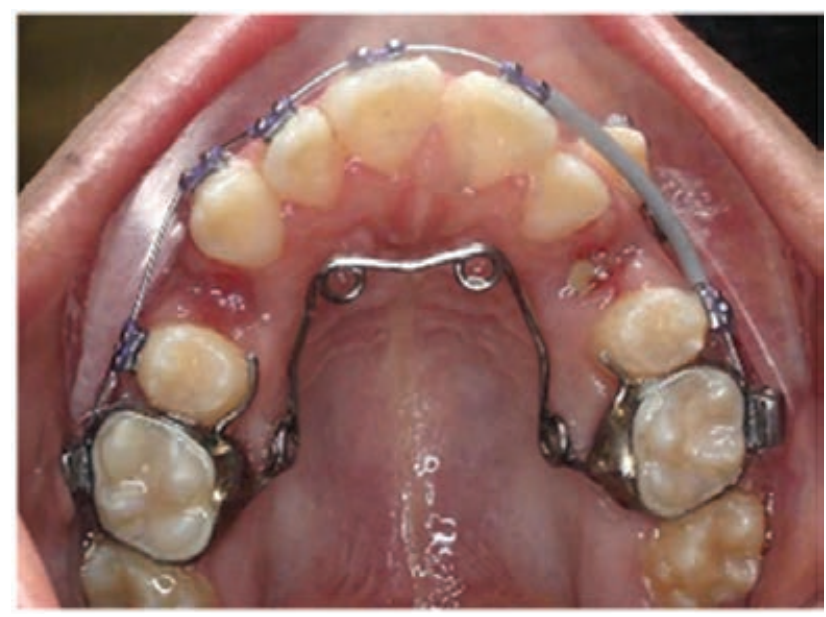

Figure 4: Retention period of quad helix appliance after maxillary expansion.

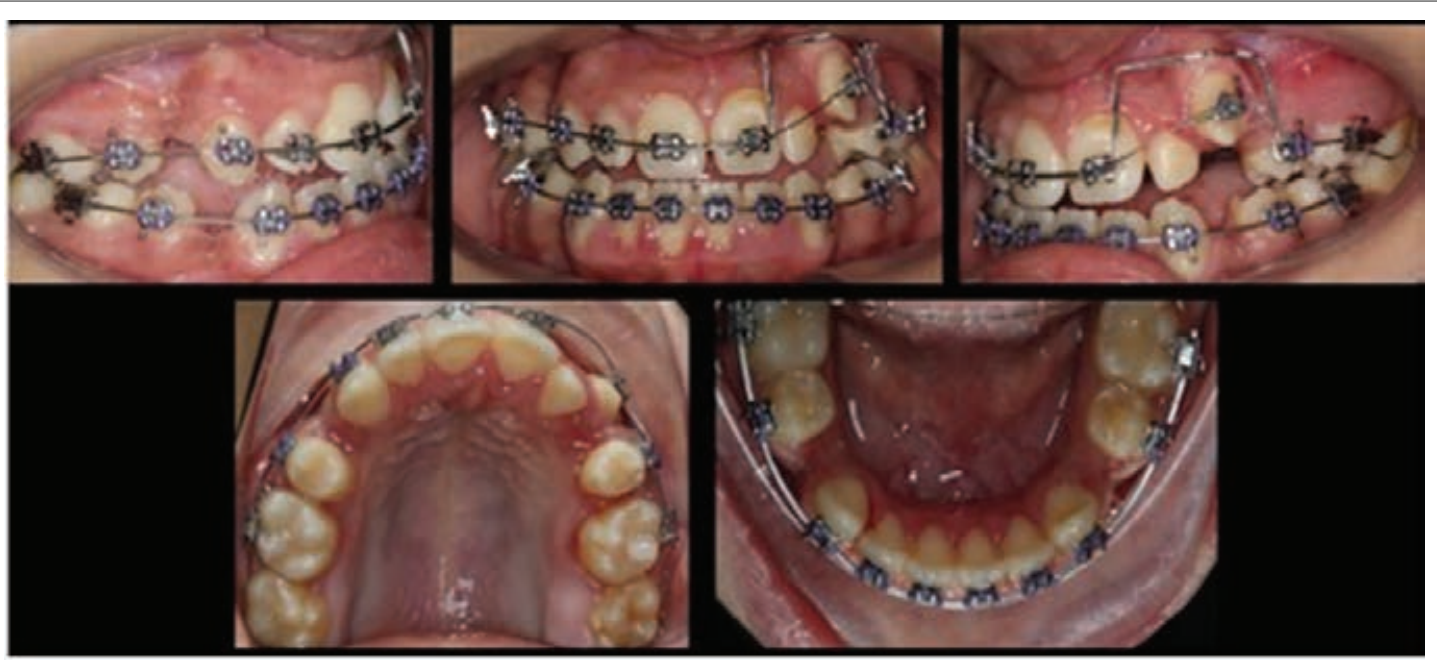

Figure 5: Piggy back technique with $0.014 \mathrm{NiTi}$ arch-wire and $0.018 \mathrm{SS}$. 
3 months for retention purposes of transverse relationship and to prevent collapsing of the upper arch. Then, the upper and lower arches were bonded by upper and lower pre-adjusted edge-wise fixed appliances with Roth prescription and 0.022 " $\times 0.028$ " slot size brackets. An initial upper $0.014 \mathrm{NiTi}$ arch-wire was also used for initial leveling and aligning. The upper left lateral incisors were bypassed to prevent the risk of tipping its root toward the upper left canine. After the expansion of the upper arch, retraction of upper left canine away from upper left lateral incisor and initial leveling and alignment, the piggyback technique was used to engage the upper left canineby 0.014 NiTi arch-wire and 0.018 SS (Figure 5). The anchorage in this case was planned and designed according to the demand of molar movement in each quadrant. In the upper and lower right sides, the anchorage was planned to be moderate anchorage and the anterior and posterior dental units were reciprocally moved toward each other in equal amount. Half of the space of the extracted $1^{\text {st }}$ premolar was closed by the forward movement of posterior teeth and the other half was closed by retraction of anterior teeth and utilized to fix upper and lower dental midlines. In the upper left side, the anchorage demand was very high because of the ectopically erupted upper left canine. The mini screw was used in addition to a segmented T-loop to retract the upper left canine without jeopardizing the anterior-posterior position of the upper left $1^{\text {st }}$ molar. Once the upper left canine was retracted and brought in the space of the extracted upper left $1^{\text {st }}$ premolar, the anchorage was burned and the amount of residual space was closed by the forward movement of the upper left $1^{\text {st }}$ molar. This was achieved by Class III elastics. On the lower-left side, the anchorage demand was balanced with the upper dental units by using Class III elastic to achieve more backward movement of anterior dental units than forwarding movement of the posterior dental units. Also, Class II elastic was used in the right side control yaw effect of Class III elastic on the left side (Figure 6). The upper and lower 2nd molars were well aligned and in proper occlusion. Also, since the nature of orthodontic biomechanics are in general extrusive movements, the $2^{\text {nd }}$ molars were not included in the treatment to minimize the risk of open bite. After the final alignment, space closure was achieved. Finally, elastics were applied for the settling of teeth.

\section{Retention plan}

The retention for this case was planned to be upper and lower fixed retainers extended from canine to canine. Upper \& lower wraparound retainers were also given to the patient to prevent the opening of extraction space.

\section{Treatment results}

Adequate facial proportions were obtained at the end of treatment with a relatively symmetrical face. The facial photographs showed a pleasant smile. A good overjet and overbite were obtained with a Class-I molar and canine relationship. The patient ends up with wellaligned upper and lower teeth. The upper and lower midlines were coincident to each other and both of them were centered to the patient's facial midline at the end of treatment (Figure 7).

\section{Discussion}

Successful orthodontic treatment was achieved in this case, which may not have been possible without the use of temporary anchorage devices as a means of absolute anchorage. Furthermore, using these devices prevented the potential adverse effects of conventional orthodontics, such as loss of anchorage and incomplete correction of intra- and inter-arch alignment problems. A titanium mini-screw was used to overcome these problems. The maxillary canine is the most commonly involved tooth in ectopic eruptions and transpositions. Shapira and Kuftinec explained this high incidence by the canines having the longest period of development and the longest path of eruption [7]. According to Yilmaz HH, et al., the most commonly seen transposition occurs with the maxillary canine-lateral incisor rather than the maxillary canine-first premolar [8]. Temporary anchorage devices have significant advantages, but their placement is sensitive to the technique and carries some risks. Potential complications include root injuries, loss of tooth vitality, osteosclerosis, and dentoalveolar ankylosis $[9,10]$. Furthermore, there is a chance that the nerve will be injured during placement in the maxillary palatal slope, where the greater palatine foramen is located $[11,12]$.

\section{Conclusions}

Mini-screw anchorage has various advantages over conventional mechanics. The treatment is simple and comfortable for both the patient and orthodontist, and there is no need for additional laboratory work. Furthermore, there is no loss of anchorage, so the entire treatment time can be shortened.

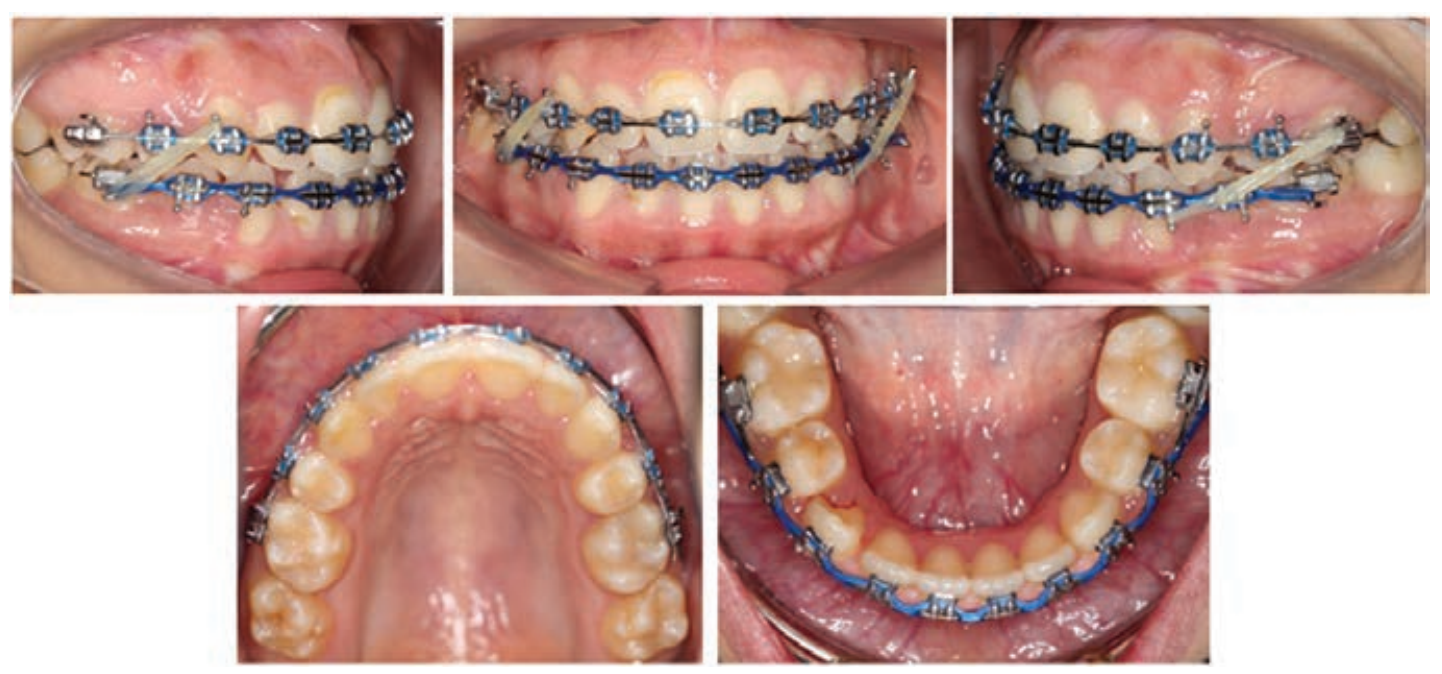

Figure 6: Space closer stage and utilization of elastic to control anchorage units. 

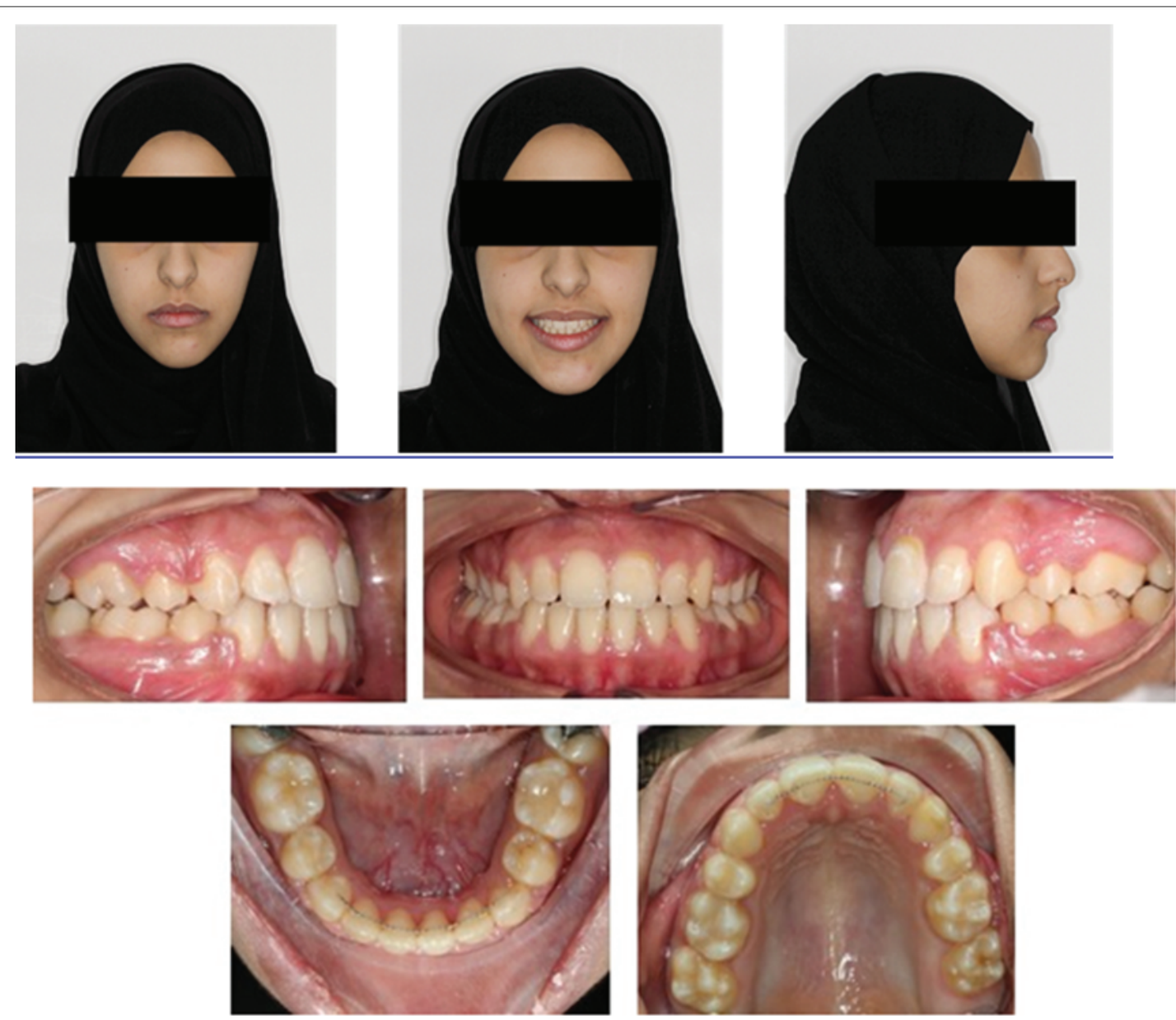

Figure 7: Post-treatment intra-oral and extra-oral photographs.

\section{References}

1. Portela MB, Sanchez AL, Gleiser R (2003) Bilateral distal ectopic eruption of the permanent mandibular central incisors: A case report. Quintessence Int 34: 131-134.

2. Gugnani N, Pandit IK, Gupta M, Gugnani S, Vishnoi A, et al. (2017) Ectopic eruption of maxillary central incisor through abnormally thickened labial frenum: An unusual presentation. J Indian Soc Pedod Prev Dent 35: 94-97.

3. Costa A, Raffaini M, Melsen B (1998) Mini-screws as orthodontic anchorage: a preliminary report. Int J Adult Orthodon Orthognath Surg 13: 201-209.

4. Kuroda S, Katayama A, Takano-Yamamoto T (2004) Severe 51 anterior open-bite case treated using titanium screw anchorage. Angle Orthod. 74: 558-567.

5. Kuroda S, Sugawara Y, Deguchi T, Kyung HM, Takano-Yamamoto T (2007) Clinical use of mini-screw implant as orthodontic anchorage: success rate and postoperative discomfort. Am J Orthod Dentofacial Orthop 131: 9-15.

6. Roberts WE, Nelson CL, Goodacre CJ (1994) Rigid implant anchorage to close a mandibular first molar extraction site. J Clin Orthod 28: 693-704.

7. Shapira Y, Kuftinec MM (1989) Tooth transposition-A review of the literature and treatment considerations. Angle Orthod 59: 271-276.
8. Yilmaz HH, Türkkahraman H, Sayin MÖ (2005) Prevalence of tooth transpositions and associated dental anomalies in a Turkish population. Dentomaxillofac Radiol 34: 32-35.

9. Asscherickx K, Vannet BV, Wehrbein H, Sabzevar MM (2005) Root repair after injury from mini-screw. Clin Oral Implants Res 16: 575578.

10. Mine K, Kanno Z, Muramoto T, Soma K (2005) Occlusal forces promote periodontal healing of transplanted teeth and prevent dentoalveolar ankylosis: an experimental study in rats. Angle Orthod 75: 637-644.

11. Sujatha N, Manjunath KY, Balasubramanyam V (2005) Variations of the location of the greater palatine foramina in dry human skulls. Indian J Dent Res 16: 99-102.

12. Wang TM, Kuo KJ, Shih C, Ho LL, Liu JC (1988) Assessment of the relative locations of the greater palatine foramen in adult Chinese skulls. Acta Anat (Basel) 132: 182-186. 